\title{
VAB-10 spectraplakin acts in cell and nuclear migration in Caenorhabditis elegans
}

\author{
Hon-Song Kim ${ }^{1,2}$, Ryoko Murakami ${ }^{2,3}$, Sophie Quintin ${ }^{4}$, Masataka Mori ${ }^{1}$, Kiyotaka Ohkura², \\ Katsuyuki K. Tamai ${ }^{2}$, Michel Labouesse ${ }^{4}$, Hiroshi Sakamoto ${ }^{3}$ and Kiyoji Nishiwaki ${ }^{1,2, *}$
}

\begin{abstract}
SUMMARY
Cytoskeletal regulation is important in cell migration. The Caenorhabditis elegans gonadal distal tip cells (DTCs) offer a simple model with which to investigate the mechanism of cell migration in organogenesis. Here, we report that one of the spectraplakin isoforms, VAB-10B1, plays an essential role in cell and nuclear migration of DTCs by regulating the actin and microtubule (MT) cytoskeleton. In the vab-10(tk27) mutant, which lacks VAB-10B1, alignment of filamentous (F)-actin and MTs was weakly and severely disorganized, respectively, which resulted in a failure to translocate the DTC nucleus and a premature termination of DTC migration. An MT growing-tip marker, EBP-2-GFP, revealed that polarized outgrowth of MTs towards the nuclei of migrating DTCs was strikingly impaired in $t k 27$ animals. A vab-10 mini-gene encoding only the actin- and MT-binding domains significantly rescued the gonadal defects, suggesting that $\mathrm{VAB}-10 \mathrm{~B} 1$ has a role in linking actin and MT filaments. These results suggest that VAB-10B1/spectraplakin regulates the polarized alignment of MTs, possibly by linking F-actin and MTs, which enables normal nuclear translocation and cell migration of DTCs.
\end{abstract}

KEY WORDS: Spectraplakin, Cytoskeleton, Cell and nuclear migration

\section{INTRODUCTION}

Morphogenesis by extension and branching of epithelial tubes is an essential event in organ formation in animals. The migration of epithelial tubes is often controlled by a cell or a group of cells at the tip of the growing tubes ( $\mathrm{Lu}$ and Werb, 2008). For example, in Drosophila melanogaster embryos, the tracheal tip cells lead the stalk cells as they invaginate from an epithelial sac and form a primary branch, which then gives rise to secondary and terminal branches that also have tip cells (Caussinus et al., 2008). In the kidney, the ureteric bud tip cells are induced by glial cell linederived neurotrophic factor and contribute to growth direction and branching of the ureteric buds (Sainio et al., 1997; Shakya et al., 2005). However, how actin and microtubule cytoskeletons are remodeled during epithelial tube extension remains poorly understood.

We explore this issue by studying the growth of the gonad in $C$. elegans. The gonad is made of two U-shaped gonad arms (epithelial tubes), which are produced by the directionally regulated migration of the gonadal leader cells, known as distal tip cells (DTCs), during larval development (Fig. 1A). Although the gonad arms do not branch, gonad development in C. elegans offers a simple model for studying the tip cell-dependent spatial and temporal regulation of epithelial morphogenesis.

There is growing evidence that spectraplakins, which are cytoskeleton crosslinkers, are involved in morphogenetic processes. These big proteins share features with members of both the spectrin

\footnotetext{
'Department of Bioscience, Kwansei Gakuin University, 2-1 Gakuen, Sanda 6691337, Japan. ${ }^{2}$ RIKEN Center for Developmental Biology, Chuo-ku, Kobe 650-0047, Japan. ${ }^{3}$ Department of Biology, Graduate School of Science, Kobe University, 1-1 Rokkodaicho, Nada-ku, Kobe 657-8501, Japan. ${ }^{4}$ Development and Stem Cells program, IGBMC, CNRS UMR7104/INSERM U. 964//Université de Strasbourg, Illkirch, Cedex F-67404, France.

*Author for correspondence (nishiwaki@kwansei.ac.jp)
}

and plakin superfamilies (Karakesisoglou et al., 2000; Leung et al., 1999; Sun et al., 2001). The spectraplakin family comprises BPAG1 and MACF/ACF7 in mammals, Kakapo/Shot in flies and VAB-10 in C. elegans (Roper et al., 2002; Sonnenberg and Liem, 2007). Spectraplakins have roles in cell migration in vivo (Goryunov et al., 2010; Guo et al., 1995) and regulate the cytoskeletal network (Dalpe et al., 1998; Kodama et al., 2003; Sanchez-Soriano et al., 2009). In endodermal cells from the $A C F 7$ knockout mouse, MTs do not grow along actin bundles and are not tethered to the actin cortex, resulting in defective migration in response to wounding. $A C F 7$-null epidermal keratinocytes also impair targeting of MTs along F-actin to focal adhesions, resulting in migration defects (Wu et al., 2008). Accumulating evidence suggests a role for spectraplakins that involves several morphogenetic processes. Axon extension in Drosophila requires the presence of binding sites for F-actin and MTs in the same Shot molecule, suggesting that Shot crosslinks F-actin and MTs, rather than mediating functionally independent interactions with F-actin and with MTs (Lee and Kolodziej, 2002a). Shot also organizes the apical accumulation of F-actin and MTs in tracheal cells that is required for lumen formation during tracheal tube fusion. In this case, however, Shot interacts with F-actin and with MTs in a functionally redundant manner, rather than acting as an MT-actin crosslinker (Lee and Kolodziej, 2002b). Thus, the function of spectraplakin in morphogenesis may differ depending on the developmental context.

In C. elegans, vab-10 was identified as the sole spectraplakin gene, which encodes several protein isoforms with distinct functions (Bosher et al., 2003). By alternative splicing, vab-10 generates two major classes of isoforms, VAB-10A and VAB-10B. VAB-10A consists of the actin-binding domain (ABD), which is followed by a plakin domain and a series of plectin repeats that are preceded by a short coiled-coil domain. By contrast, VAB-10B contains the $\mathrm{ABD}$, plakin domain, spectrin repeats and the Gas2related domain or microtubule-binding domain (MTBD). Therefore, VAB-10A is similar in domain organization to BPAG1e 

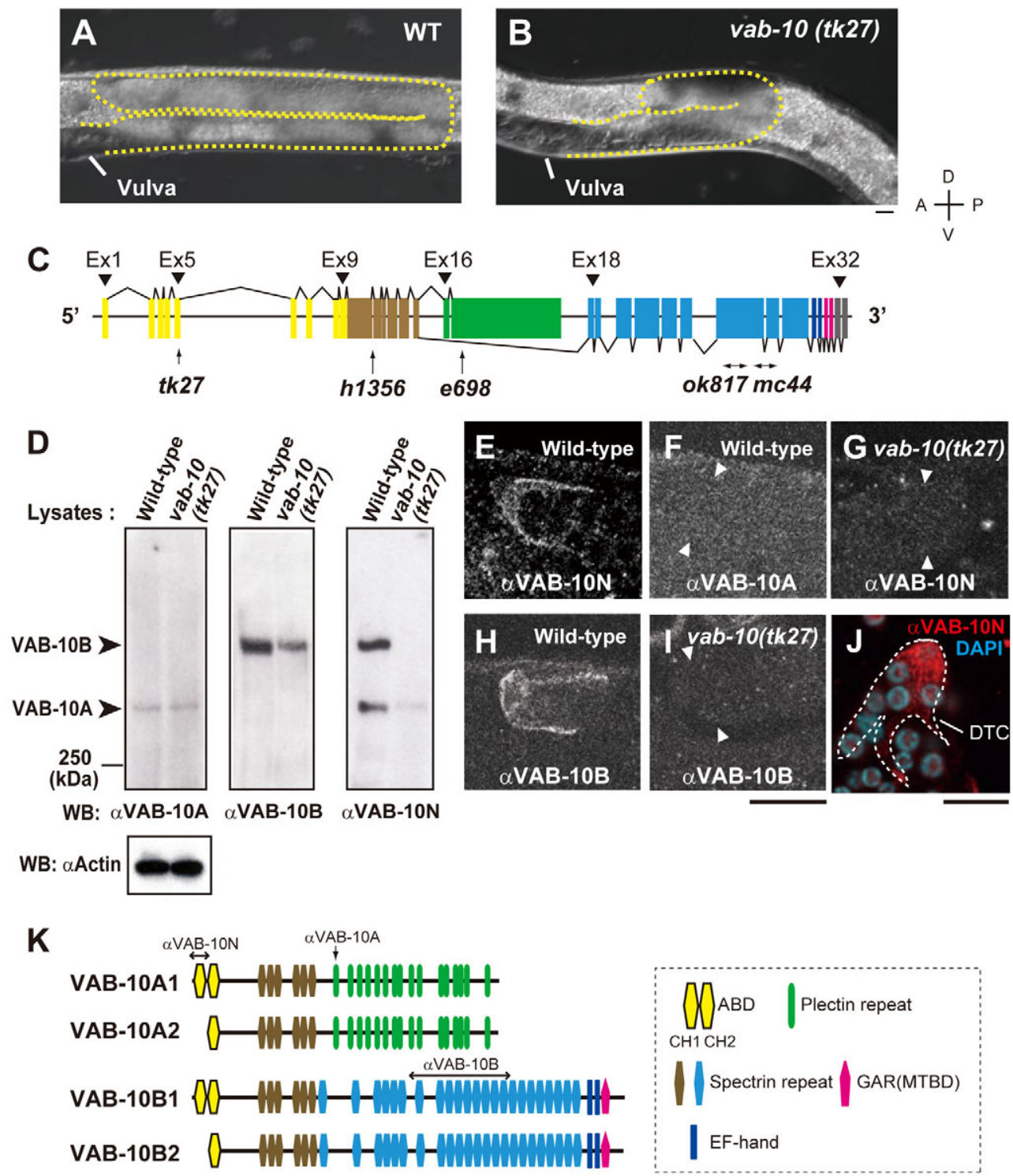

Fig. 1. Expression of VAB-10 isoforms in wild-type and vab-10(tk27) mutant animals. (A,B) Nomarski images of posterior gonad arms of wild-type (A) and vab-10(tk27) mutant (B) hermaphrodites. Gonads are shown by broken outlines. Scale bar: $10 \mu \mathrm{m}$. (C) vab-10 gene and positions of mutations (Bosher et al., 2003). The vab-10 gene consists of 32 exons. Exons common to VAB-10A and VAB-10B are shown in yellow and brown. VAB-10A-specific exons are shown in green. The exons downstream from exon 18 are VAB-10B specific. The color codes correspond to those in K. tk27 and e698 are point mutations Q148Stop (CAA to TAA) in exon 5 and P1666S (CCA to TCA) in exon 17, respectively. ok817 and mc44 are deletions in exon 24 (of nucleotides 37145-39188) and in exons 24 and 25 (of nucleotides 39204-40235), respectively, which potentially truncate VAB-10B isoforms. h1356 is a G-to-A transition in the GT consensus donor splice site of intron 10, which leads to a premature stop codon downstream. (D) Wild-type and vab-10(tk27) mutant lysates containing $20 \mu \mathrm{g}$ of protein were immunoblotted with anti-VAB-10A (upper left panel), anti-VAB-10B (upper middle) and anti-VAB-10N (upper right). An anti-actin immunoblot is shown as a loading control: left, wild type; right, vab10(tk27). The protein levels are quantified in Table S1 in the supplementary material. Similar results were obtained in three independent experiments. (E-J) VAB-10 expression in DTCs. Both anti-VAB-10N and anti-VAB-10B detected signals in wild-type DTCs (E,H) but not in vab10(tk27) DTCs (G,I). The same specimen in E was not stained by anti-VAB-10A (F). DTCs (not stained) are shown between the two arrowheads $(F, G, I)$. (J) Dissected gonads were fixed and stained with anti-VAB-10N. Anti-VAB-10N detected discontinuous filamentous structures (red) in DTCs. Nuclei are stained with DAPI (blue). Scale bars: $10 \mu \mathrm{m}$. (K) Predicted structures of VAB-10A and VAB-10B isoforms. The antigenic regions for antiVAB-10 antibodies are indicated. The VAB-10A2 and B2 isoforms are not listed in WormBase. This could be because all the CDNA clones that have been sequenced are partial sequences and because analysis based on RT-PCR amplification allows identification only of the longer isoforms VAB$10 \mathrm{~A} 1$ and B1.

and plectin, whereas VAB-10B has the same basic structure as MACF/ACF7 and BPAG1a (Roper et al., 2002). VAB-10A and VAB-10B isoforms show distinct distributions and functions in the epidermis. Loss of VAB-10A impairs the integrity of fibrous organelles, which are related to hemidesmosomes, leading to epidermal detachment from the cuticle and muscles. By contrast, an absence of VAB-10B leads to increases in epidermal thickness during embryonic morphogenesis (Bosher et al., 2003). Thus, $C$. elegans spectraplakins protect epidermal cells from external forces exerted by muscles and from internal forces resulting from cell 
shape changes that occur in the epidermis during embryogenesis (Bosher et al., 2003). However, the roles of $C$. elegans spectraplakins in morphogenetic processes in postembryonic development remain to be explored.

Here, we report a role for VAB-10 spectraplakins in linking Factin and MTs that is essential for the migration of DTCs and their nuclei during the formation of the U-shaped gonads of $C$. elegans. We isolated a loss-of-function mutant, vab-10(tk27), which exhibits abnormal gonadogenesis accompanied by a nuclear translocation defect of DTCs. Our studies suggest that abnormal gonad formation in the vab-10(tk27) mutant results from defective organization of the MT cytoskeleton within DTCs that is due to the absence of a novel VAB-10B isoform called VAB-10B1. We propose that $\mathrm{VAB}-10 \mathrm{~B} 1$ spectraplakin functions as a regulator for cytoskeletal organization within DTCs, and that its crosslinking activity of F-actin and MTs is essential for cell and nuclear migration of DTCs during gonadogenesis.

\section{MATERIALS AND METHODS}

Strains and culture conditions

Culture and handling of C. elegans were as described previously (Brenner, 1974). The following mutants were used: $\mathrm{N} 2$ (wild type), dpy-5(e61), unc75(e950), unc-101 (ml) and unc-119(e2498) (Maduro and Pilgrim, 1995); $v a b-10(t k 27)$ (this work), vab-10(e698), vab-10(mc44) and vab-10(h1356) (Bosher et al., 2003); and vab-10(ok817) (International C. elegans Gene Knockout Consortium).

\section{Isolation of the vab-10(tk27) mutant and genetic mapping}

The $t k 27$ mutant was generated by ethyl methanesulfonate mutagenesis. The mutant was outcrossed four times against the wild-type N2 strain. Single nucleotide-polymorphism mapping placed the mutation between genomic clones ZK1151 and Y47H9C. Sequence analysis revealed a mutation in the vab-10 gene.

\section{Plasmid construction}

To construct Ex1-5::Venus and Ex6-9::Venus, two genomic regions of vab10 , from -3336 to +4305 and from +4306 to +14708 , respectively (relative to the adenine of the initiation codon), were amplified by PCR and ligated to the Venus vector (Nagai et al., 2002), which was a gift from Dr Takeshi Ishihara (Kyushu University, Japan). For construction of mig$24 p:: G F P: \because$ Moesin, the C-terminal tail of Drosophila moesin, which encodes the ABD (280 amino acids) (Edwards et al., 1997), was fused with two copies of the gene encoding GFP, which were tandemly joined, and was placed downstream of the mig-24 promoter. The cDNAs corresponding to VAB-10B ABD (exons $1-9,+1$ to +1218 ) and MTBD (exons $29-32,+14134$ to +14832 of the $v a b-10 B$ cDNA) were synthesized from total RNA of wild-type worms using Superscript III Reverse Transcriptase (Invitrogen). cDNAs were used for creating mig$24 p:: A B D::$ Venus and mig-24p::ABD::MTBD::Venus plasmids. For construction of mig-24p::MTBD::Venus, the genomic fragment of exons 25-32 (+40134 to +44302) was used. For construction of mig-24p::ebp$2:: G F P$, the full-length $e b p-2$ genomic DNA was amplified by PCR as described by Srayko et al. (Srayko et al., 2005) (primer sequences upon request), fused with GFP and placed downstream of the mig-24 promoter.

\section{Production of transgenic animals}

DNA mixtures were first injected into unc-119(e2498) animals (Mello et al., 1991), and the transgenic arrays were transferred to vab-10(tk27) mutants by mating. Ex1-5::Venus and Ex6-9::Venus plasmids were injected at $10 \mu \mathrm{g} / \mathrm{ml}$ with $160 \mu \mathrm{g} / \mathrm{ml}$ of pBSII KS(-) and $30 \mu \mathrm{g} / \mathrm{ml}$ of $u n c-119^{+}$ plasmid pDP\#MM016B (Maduro and Pilgrim, 1995). In the following experiments, if the DNA concentration was $<150 \mu \mathrm{g} / \mathrm{ml}$ without pBSII $\mathrm{KS}(-)$, then pBSII $\mathrm{KS}(-)$ plasmid was included to bring the final DNA concentration to $150 \mu \mathrm{g} / \mathrm{ml}$. mig-24p::GFP::Moesin plasmid was injected at $10 \mu \mathrm{g} / \mathrm{ml}$ with $20 \mu \mathrm{g} / \mathrm{ml}$ of $u n c-119^{+}$plasmid. mig-24p::ABD::Venus, mig-24p::MTBD::Venus and mig-24p::ABD::MTBD::Venus plasmids were individually injected at $10 \mu \mathrm{g} / \mathrm{ml}$ with $30 \mu \mathrm{g} / \mathrm{ml}$ of $u n c-119^{+}$plasmid. To generate transgenic animals that co-expressed the ABD and MTBD of VAB-10B1, mig-24p::ABD::Venus and mig-24p::MTBD::Venus were coinjected at $10 \mu \mathrm{g} / \mathrm{ml}$ with $30 \mu \mathrm{g} / \mathrm{ml}$ of $u n c-119^{+}$plasmid. To assess the rescue activity of each domain of VAB-10B1, each transgenic array was transferred to the $v a b-10(t k 27)$ mutant by mating. The transgenic animals that expressed EBP-2-GFP were obtained by injecting mig-24p::ebp$2 \because \because G F P$ and $u n c-119^{+}$plasmids at $10 \mu \mathrm{g} / \mathrm{ml}$ and $30 \mu \mathrm{g} / \mathrm{ml}$, respectively. More than three independent transgenic lines with similar phenotypes were obtained. Transgenic lines for mig-24p::GFP::Moesin, mig$24 p:: M T B D::$ Venus and mig-24p::ebp-2::GFP used in this work had no deleterious effects on DTC migration (see Fig. S1 in the supplementary material).

\section{Production of antibodies}

The N-terminal half of the ABD (M1-Q170) was expressed as a GST fusion protein in Escherichia coli and was used to immunize rabbits. The antibodies were affinity purified.

\section{Western blot analysis}

Mixed stage worm extracts ( $20 \mu \mathrm{g}$ protein) were separated on $4.2 \%$ SDSpolyacrylamide gels and immunoblotted with MH5 (1:50; Developmental Studies Hybridoma Bank), K22 (1:1000), anti-VAB-10N (1:500), anti-actin clone C4 (1:1000; MP Biochemicals).

\section{Immunohistochemistry}

Animals were fixed as described (Finney and Ruvkun, 1990). After blocking with 5\% normal donkey serum (Jackson ImmunoResearch) in phosphate-buffered saline (PBS), samples were incubated with the following primary antibodies in PBS containing 5\% normal donkey serum and $0.5 \%$ Triton $\mathrm{X}-100$ at $4{ }^{\circ} \mathrm{C}$ overnight: anti-VAB-10A (1:50), anti-VAB10N (1:200), anti-VAB-10B (1:500), anti-GFP IgG (3E6, 1:200; Molecular Probes) and anti- $\alpha$-tubulin (DM1A, 1:200; Sigma). Samples were incubated with appropriate secondary antibodies conjugated with either rhodamine or fluorescein isothiocyanate (Jackson ImmunoResearch) at room temperature for 4 hours. The dissected gonads were fixed with methanol at $-20^{\circ} \mathrm{C}$ for 10 minutes. Blocking and staining were as described above.

\section{Microscopy}

Nomarski and fluorescence microscopy was performed as described (Kubota et al., 2006). The laser-scanning confocal microscope (LSM5 PASCAL version 3.5; Zeiss) with a $100 \times$ objective was used for live imaging. The confocal images were obtained with a $0.5 \mu \mathrm{m} z$-series. Movies of EBP-2-GFP signals were acquired with a spinning-disk confocal scan head (CSU-X1; Yokogawa) that was mounted on a Zeiss Imager M2 microscope equipped with an EM-CCD camera (ImageEM; Hamamatsu Photonics). Images were acquired in single confocal planes at a rate of 200 mseconds/frame.

\section{Quantitative analysis}

The nuclear translocation defects of DTCs were scored with Nomarski microscopy. For quantification of the polarized localization of MTBDVenus, individual pixel areas at the trailing and leading ends of the DTCs were measured using Image $J$ software. The data for the direction of actin filaments and of comet migration were tracked and collected using the software 'Region Measurements' of MetaMorph (Molecular Devices). The directional distribution was evaluated and displayed using Rose diagrams (Rose 2.1.0; Todd A. Thompson, http://mypage.iu.edu/ tthomps/programs/ home.htm) as described previously (Harumoto et al., 2010).

\section{RESULTS \\ C. elegans spectraplakin VAB-10 is required for gonadal leader cell migration}

The $C$. elegans $\mathrm{U}$-shaped hermaphrodite gonad is generated by the migration of two DTCs (Fig. 1A). The two DTCs, which form at the tips of the gonad primordium, migrate both anteriorly and posteriorly during second larval stage (L2) and early L3. The DTCs turn dorsally during mid-L3 and migrate across the lateral 
hypodermis. They turn again over the dorsal muscle around the time of the L3 molt and subsequently migrate towards each other along the dorsal muscle, thereby generating symmetrical U-shaped gonad arms with respect to the dorsoventral axis at the center of the body. We isolated a mutant, $t k 27$, that exhibited shortened gonad arms when compared with those in wild-type animals because the migration rate of DTCs was globally slower and the DTCs were arrested after the second turn (Fig. 1A,B). We measured the timing of the DTC turn relative to the stage of vulval development (see Fig. S2A,B in the supplementary material). The vulval precursor cell P6.p undergoes three rounds of cell division and produces eight descendants that constitute the vulva. In the wild-type animals, the DTCs always made the first turn over the ventral muscle at the four-P6.p cell stage. By contrast, $65 \%$ of DTCs made the first turn at the eight-P6.p cell stage in $t k 27$ animals. Although the second turn occurred most often at the four-P6.p cell stage in wild type, the DTCs in $t k 27$ animals always made the second turn at the eightP6.p cell stage. Thus, the DTCs migrate more slowly in the $t k 27$ mutant than they do in wild type. Although $t k 27$ hermaphrodites proliferated as homozygotes with small broods, males were sterile (data not shown).

We found that the $t k 27$ mutation was a nonsense mutation in the fifth exon of the vab-10 gene, which encodes spectraplakin isoforms, potential cytoskeletal linker proteins in $C$. elegans (Bosher et al., 2003) (Fig. 1C). Previously isolated vab-10 mutations cause embryonic lethality with severe epidermal morphogenetic defects or abnormal head morphogenesis (Bosher et al., 2003). Although the $t k 27$ mutation changes glutamine 148 to a stop codon and is the most upstream mutation among the previously reported vab-10 mutant alleles, animals with this mutation exhibited defects in gonad development without any lethality or head deformation. Exons 1-9 of vab-10 encode an ABD (Bosher et al., 2003), and the $t k 27$ mutation is likely to result in the truncation of the polypeptide after the N-terminal half of the ABD.

In $C$. elegans, the single spectraplakin gene $v a b-10$ produces mainly two isoform classes, VAB-10A and VAB-10B, by alternative splicing. We carried out complementation tests in combination with $v a b-10 A$-specific $e 698$ (head morphogenesis defective) or $v a b-10 B$-specific $o k 817$ and $m c 44$ (embryonic or early larval lethal) alleles. e698/tk27 heteroallelic animals were fully wild type, indicating that they complemented each other. ok817/tk27 and mc44/tk27 heterozygotes showed a defective gonad phenotype similar to that seen in $t k 27$ homozygotes but not a lethal phenotype, indicating that $t k 27$ complemented the lethality of ok817and $m c 44$, whereas those alleles failed to complement the migration defect of $t k 27 . t k 27$ also complemented the embryonic lethality of the null allele $h 1356$, and $t k 27 / h 1356$ animals grew to become sterile adults with $t k 27$-like DTC migration defects. The sterility could have resulted from the synergistic effect of $h 1356$, which reduces the $v a b-10$ gene dose (for all the isoforms), and $t k 27$, which reduces the gene dose for a specific set of isoforms. Thus, $t k 27$ mutants are likely to be specifically defective in $v a b$ $10 B$ function in gonadogenesis.

\section{vab-10(tk27) animals lack subsets of the VAB-10A and VAB-10B isoforms}

Using MH5 and K22 antibodies, which recognize VAB-10A and VAB-10B isoforms, respectively (Bosher et al., 2003), we examined the patterns of expression of VAB-10 proteins. Herein, we refer to MH5 and K22 as anti-VAB-10A and anti-VAB-10B, respectively (Fig. 1K). Anti-VAB-10A detected similar-sized proteins in both wild-type and mutant lysates on western blots (Fig.
1D, upper left). Anti-VAB-10B detected VAB-10B isoform(s) of $\sim 500 \mathrm{kDa}$ in the wild-type lysates (Fig. 1D, upper middle). Although it detected a similar-sized band in vab-10(tk27) lysates, the signal was apparently reduced when compared with that in the wild-type lysates. These results suggest that a subset of the VAB$10 \mathrm{~B}$ isoforms is affected by $t k 27$. We raised a polyclonal antibody against the N-terminal 170 -amino acid peptide that is encoded by exons 1-5 (anti-VAB-10N). Anti-VAB-10N specifically detected a band of $\sim 500 \mathrm{kDa}$ and a smaller-sized band with a molecular mass similar to that of VAB-10A in the wild-type lysates, but it detected only a very faint band near VAB-10A in vab-10(tk27) lysates (Fig. $1 \mathrm{D}$, upper right). Although the molecular identity of the protein corresponding to this faint band is not clear, it could be an unidentified VAB-10 variant having the N-terminal half of the $\mathrm{ABD}$ that is expressed weakly even in the $t k 27$ mutants. Alternatively, the faint band could be of non-specific binding of anti-VAB-10N. These results suggest that $t k 27$ is mostly deficient for VAB-10A and VAB-10B isoforms containing the N-terminal peptide.

We examined the expression of VAB-10 proteins in DTCs. We found that both anti-VAB-10N and anti-VAB-10B detected signals in wild-type DTCs, but they failed to do so in $t k 27$ DTCs (Fig. 1E,G,H,I). Anti-VAB-10A failed to reveal signals even in wild-type DTCs (Fig. 1F). Although anti-VAB-10N uniformly stained the cytoplasm of DTCs in whole-mount worm preparations, it detected discontinuous filamentous structures in the dissected gonads (Fig. $1 \mathrm{~J})$. These results suggest that the filamentous antigen localization may be susceptible to fixative conditions. Immunohistochemical analysis also revealed that anti-VAB-10A stained fibrous organelles in the epidermis, and anti-VAB-10B detected reciprocal circumferential bands in the epidermis both in the wild-type and in tk27 animals (see Fig. S3 in the supplementary material). Although anti-VAB-10N detected the bands that were detected by anti-VAB$10 \mathrm{~A}$ in the wild-type epidermis, it failed to detect them in $t k 27$ animals. We also detected signals in the pharynx and body wall muscle using anti-VAB-10B and anti-VAB-10N (H.-S.K. and K.N., unpublished; a detailed account of VAB-10 distribution in these tissues will be presented elsewhere).

Taken together, these results suggest that there are at least two different VAB-10A and two different VAB-10B isoforms depending on whether they have (VAB-10A1 and VAB-10B1) or do not have (VAB-10A2 and VAB-10B2) the N-terminal 170amino acid region. We showed that these two different isoforms could be expressed by two distinct promoters (see Fig. S4 in the supplementary material).

\section{DTCs dynamically change their morphology during dorsal migration}

C. elegans VAB-10B1 possesses the $\mathrm{ABD}$ and $\mathrm{MTBD}$ at its $\mathrm{N}$ and $\mathrm{C}$ terminus, respectively. To visualize the actin filaments of DTCs, we expressed a GFP fusion construct of the ABD of Drosophila Moesin (GFP-Moesin) under the control of the DTC-specific mig24 promoter (Tamai and Nishiwaki, 2007). The GFP-Moesin marker makes it possible to analyze the morphology of DTCs, probably because it highlights the cortical actin network. We analyzed the morphology of DTCs using three-dimensional reconstruction of confocal images. The DTCs migrating on the ventral body wall muscle exhibited a trapezoid morphology in the optical cross-section (Fig. 2A). When turning dorsally, they extended a single large lamellipodium into the contact site of the intestine and the lateral epidermal ridge (Fig. 2B). The DTCs further extended their lamellipodia into the contact site and began 

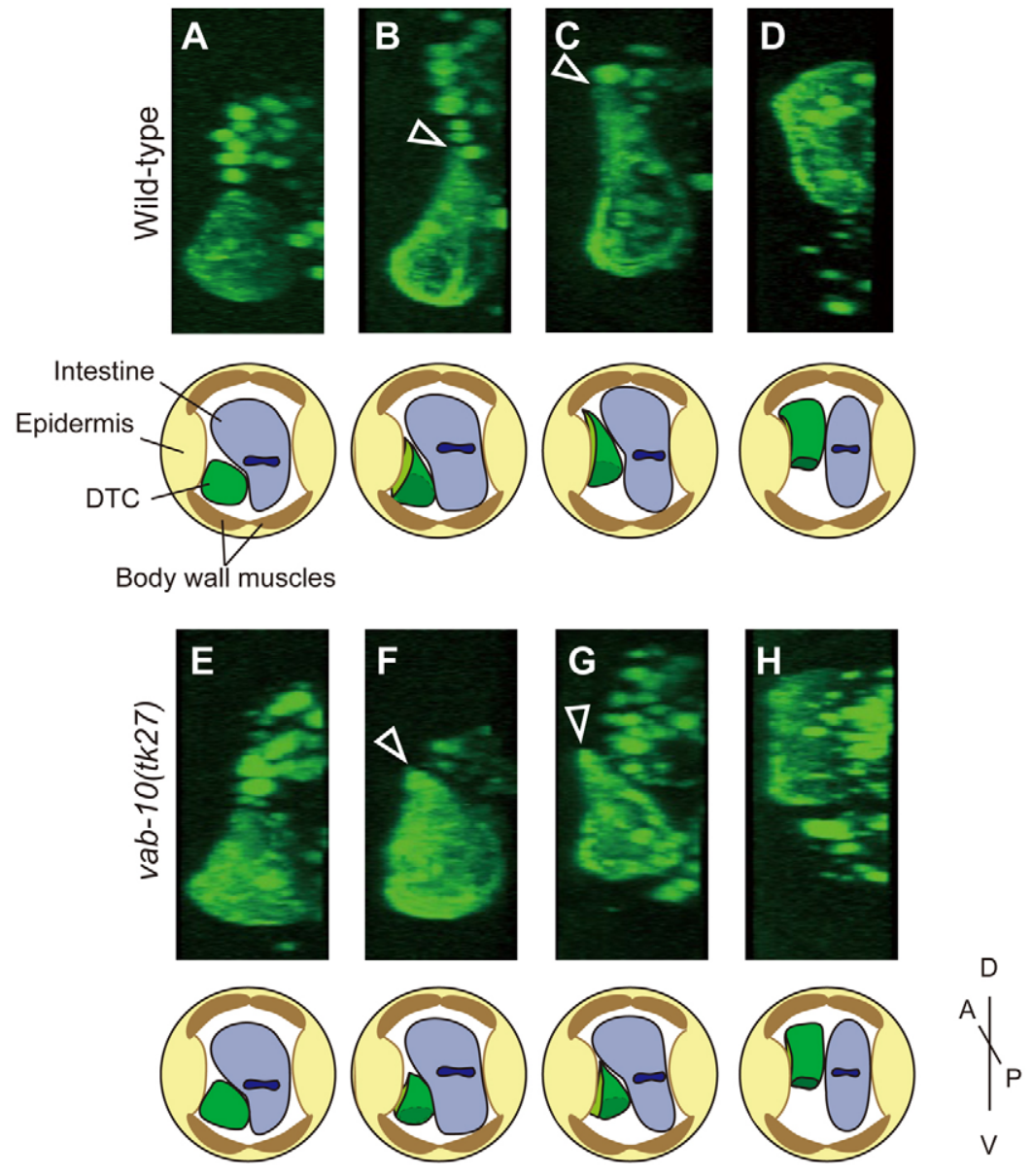

Fig. 2. z-slice images of DTCs during dorsal

migration. (A-H) Expression of GFP-Moesin was driven

by the DTC-specific mig-24 promoter in the wild type (A-

D) and in vab-10(tk27) animals (E-H). z-sections of the

three-dimensional reconstructed confocal images of posterior DTCs are shown. DTCs migrating on the ventral muscle $(A, E)$, initiating extension of a dorsal lamellipodium $(B, F)$, moving dorsally between epidermis and intestine $(C, G)$, and reaching the dorsal muscle $(D, H)$ are shown. The arrowheads indicate the lamellipodium. Schematics for each DTC are shown below the images. to migrate dorsally (Fig. 2C). After reaching the dorsal muscle, they retracted the trailing part dorsally and gradually shifted their migratory routes from the lateral epidermis to the dorsal muscle (Fig. 2D). In vab-10(tk27) animals, the migration of DTCs occurred essentially in a similar pattern, although the lamellipodial extension was not as extensive as that in the wild type (Fig. 2E-H).

\section{Loss of VAB-10B1 inhibits nuclear translocation of DTCs during their dorsal turn}

When examined from the lateral side, the wild-type DTCs exhibited a U-shaped morphology that placed the nuclei at the leading edge of the DTCs during the migration over the ventral body wall muscle (Fig. 3A). When turning dorsally, the nuclei were relocated to the dorsal side of the DTCs (Fig. 3B). Concomitantly, the edges of the concave cell surface at the trailing end began to close, enfolding a few germline nuclei (Fig. 3B). The dorsally turned DTCs were ring-shaped (Fig. 3C). At the second turn, the ring-shaped DTCs were redirected, placing the nuclei at the leading edge (Fig. 3D,E). The DTCs in vab-10(tk27) mutants were Ushaped and had nuclei at the leading edge, as did those in the wild type, until the first turn (Fig. 3F). Interestingly, however, the nuclear translocation of DTCs at the dorsal turn was suppressed in $v a b-10(t k 27)$ mutants (Fig. 3G,H,I). Although mutant DTCs were attracted dorsally, presumably by the UNC-6/netrin-dependent guidance cues (Culotti and Merz, 1998; Su et al., 2000), their nuclei stayed at the anterior or posterior ends rather than being relocated to the dorsal side of the DTCs, as in the wild type (Fig. $3 \mathrm{~K})$. After the second turn, the DTCs began to migrate over the dorsal muscle, with the nuclei remaining at the trailing end of the cells (Fig. 3J). We analyzed the nuclear translocation in the unc6(ev400)-null mutant background. Although the DTCs were always directed dorsally at the first turn in the wild type, they turned dorsally or ventrally as well as laterally in unc- 6 mutants. When we examined the unc-6 DTCs after the first turn, the nuclei were always translocated to the leading edge of the DTCs irrespective of the turning direction (see Table S2 in the supplementary material). Thus, the direction of nuclear translocation correlates with the turning direction of DTCs, which is normally controlled by the UNC-6 signal. In the unc-6(ev400); vab-10(tk27) double mutants, the DTCs also turned in various directions, but their nuclei stayed at the anterior or posterior ends in most cases, indicating loss of the link between turning direction of DTCs and translocation of their nuclei in $t k 27$ animals. As nuclear migration in embryonic hyp7 precursor cells, which subsequently fuse to form a syncytial epidermis called hyp7, has been studied (Fridolfsson and Starr, 2010; Meyerzon et al., 2009), we examined the nuclear migration in vab-10(h1356) mutants. We found that the mutants have weak hyp7 nuclear migration defects (see Fig. S5 in the supplementary material), suggesting that $v a b-10$ plays a minor role in this nuclear migration.

\section{The MT array is severely disorganized in DTCs in vab-10(tk27) animals}

To examine whether the F-actin and MTs were affected by the $v a b-10(t k 27)$ mutation, we visualized both components using fluorescent cytoskeletal markers: GFP-Moesin (described above) 


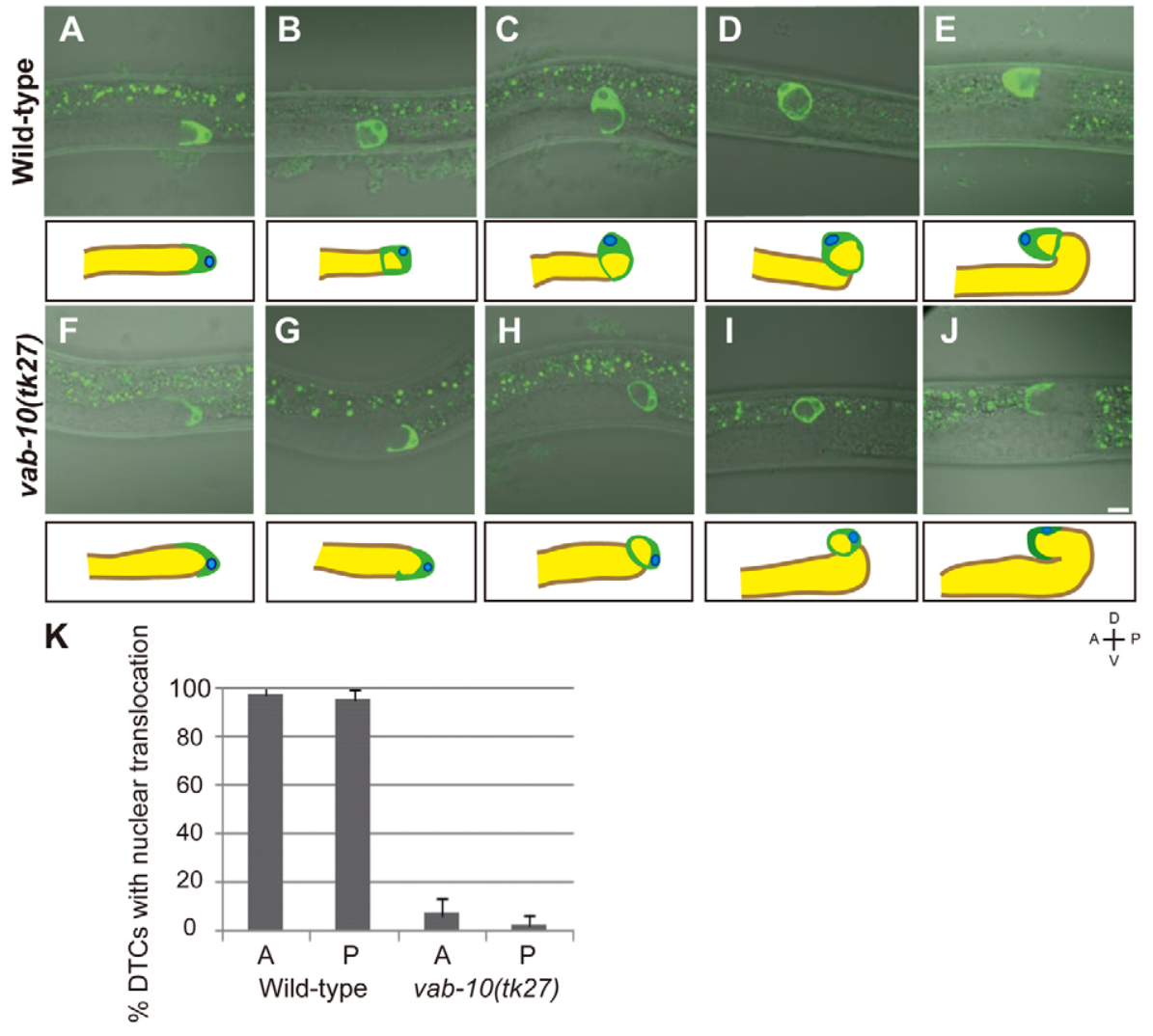

Fig. 3. Loss of VAB-10B1 inhibits nuclear translocation of DTCs during their dorsal turn. (A-J) Merged live images of Nomarski and GFP-Moesin fluorescence in wild-type (AE) and vab-10(tk27) mutant (F-J) DTCs. Each panel represents a single confocal plane in which the nucleus was detected. Single posterior gonads with DTCs migrating on the ventral muscle $(A, F)$, initiating the dorsal turn $(B, G)$, migrating dorsally $(C, H)$, reaching the dorsal muscle $(D, I)$ and after the second turn $(E, J)$ are shown. Each image is illustrated schematically in lower panels. Gonad arms, region containing germline nuclei, DTCs and DTC nuclei are shown in brown, yellow, green and blue, respectively. Anterior is leftwards and dorsal is upwards.

(K) Percentages of DTCs that undergo nuclear translocation at the first turn in wild-type and vab-10(tk27) animals. Anterior (A) and posterior (P) DTCs were scored independently. Data are shown as the mean \pm s.d. ( $n=45$ for each experiment).

for the actin cytoskeleton and VAB-10 MTBD-Venus (MTBDVenus) as a marker for the MT cytoskeleton. We found that GFPMoesin was present as filamentous structures that were often aligned along the migratory axis in DTCs (Fig. 4A-C). When DTCs expressing GFP-Moesin were exposed to Latrunculin B, an inhibitor of actin polymerization, the filamentous GFP signals were absent (see Fig. S6A-D in the supplementary material), suggesting that GFP-Moesin associates with F-actin in migrating DTCs. The network of F-actin in the mutant DTCs was similar to that in the wild-type DTCs, although the directional alignment of the filaments appeared to be somewhat perturbed (Fig. 4D-F; see Fig. S7 in the supplementary material). The F-actin at the trailing end of the DTCs was dynamically rearranged during the dorsal turning of the DTCs. Although the F-actin tended to be oriented along the anteroposterior axis before the dorsal turn (Fig. 4A), they came together to form thick bundles that surrounded the edges of the concave cell surface at the trailing end of the DTCs at the initiation of the dorsal turn (Fig. 4B). This bundling was also observed for F-actin in vab-10(tk27) animals (Fig. 4E). Because this cytoskeletal rearrangement occurs concomitantly with the enclosure of the trailing end of the DTCs, it may drive the enclosure process.

MTBD-Venus also showed filamentous localization (Fig. 4G-I). Because the MTBD-Venus signals colocalized with endogenous MTs (see Fig. S6E-G in the supplementary material), it is likely that MTBD-Venus associates with the MT cytoskeleton in DTCs. We found that MTBD-Venus showed polarized localization, as it was enriched in the trailing end of the DTCs (Fig. 4G-I). The average fluorescence intensity of the trailing end was about three times higher than that of the leading end of the DTCs in the wild type (Fig. 4M). In the vab-10(tk27) mutant, the array of MT filaments was severely disorganized, and the polarized localization of MTBD-Venus was completely lost (Fig. 4J-L). These results suggest that VAB-10B1 plays an important role in the organization of the MT cytoskeleton within migrating DTCs.

\section{Analysis of the growing (plus) ends of MTs}

In order to find out whether MT dynamics were perturbed, we tracked growing ends of MTs in migrating DTCs using the plus-end tracking protein EBP-2 fused with GFP (EBP-2-GFP) (Srayko et al., 2005 ) by time-lapse videomicroscopy. The directional distribution of the shooting comets was analyzed in DTCs migrating on the ventral muscle, making the first turn, and migrating dorsally between the intestine and the epidermis. We always observed obvious differences in the patterns of comet movements between the front (nuclear) and rear regions of the migrating DTCs (Fig. 5A,B,F; see Movies 1-12 in the supplementary material). In the wild type, the EBP-2-GFP comets in the front region revealed polarized MT growth toward the cell nucleus in the leading edge of the DTCs (Fig. 5A'), whereas those in the rear region showed growth of MTs that was for the most part random (Fig. 5B'). By contrast, we observed randomized patterns of shooting comets in both front and rear regions in the vab10(tk27) DTCs (Fig. 5C-D'), in which the numbers of comets were significantly reduced in the front region (see Table S3 in the supplementary material).

\section{VAB-10B1 mediates the interaction between actin and MT filaments that is required for nuclear migration of DTCs}

Proteins of the spectraplakin family act as linker proteins for actin and MT filaments in other organisms (Karakesisoglou et al., 2000; Lee and Kolodziej, 2002a; Wu et al., 2008). Thus, we investigated the possible role of $C$. elegans VAB-10B1 in linking F-actin with MTs. Because a construct consisting of only the N-terminal ABD 

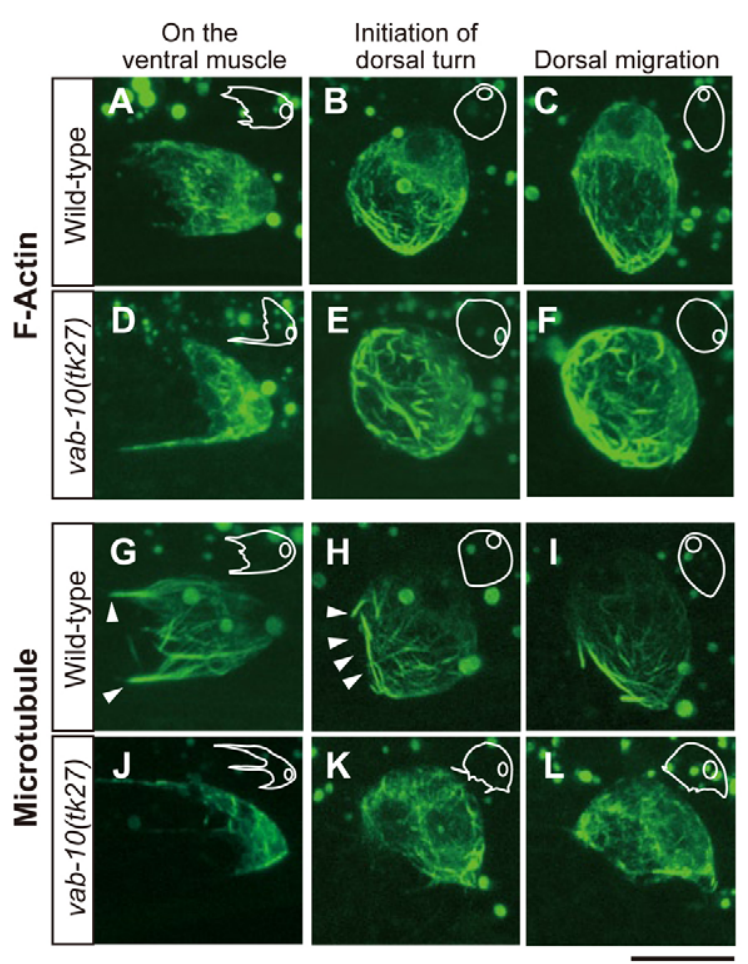

Fig. 4. Distribution of actin and MT filaments in DTCs. (A-L) Z-stack confocal images (0.5 $\mu \mathrm{m}$ intervals) of DTCs expressing GFP-Moesin (A-F) and MTBD-Venus (G-L). Wild-type (A-C,G-I) and vab-10(tk27) (D-F,J-L) DTCs are shown. Outlines of DTCs and their nuclei are illustrated schematically with white lines in each image. (M) Quantification of MTBD-Venus polarization in wildtype and vab-10(tk27) mutant animals. L2 and L3 larvae were scored. The intensity of Venus fluorescence at the trailing end was threefold $(3.08 \pm 1.62)$ higher than that at the leading end of DTCs in wild-type animals. In the vab-10(tk27) mutant, the intensity ratio was $0.845 \pm 0.43$, indicating that the polarity was lost. Data are shown as the mean \pm s.d. ( $n=14$ DTCs for each experiment). Data were analyzed with Welch's t-test; the resulting $P$ value is shown. and the C-terminal MTBD can restore radial MT organization in cultured cells that are null for ACF7 (Kodama et al., 2003; Wu et al., 2008) and can rescue axonal growth phenotypes in shot mutant Drosophila embryos (Lee and Kolodziej, 2002a), we carried out rescue experiments using several deletion constructs (Fig. 6A). All constructs used in this experiment were fused with Venus at their $\mathrm{C}$ termini and were driven by the DTC-specific mig-24 promoter. Although neither ABD-Venus nor MTBD-Venus rescued the nuclear translocation defect in $v a b-10(t k 27)$ mutants, expression of ABD-MTBD-Venus only in DTCs did efficiently rescue this defect (Fig. 6B). ABD-MTBD-Venus restored not only the nuclear translocation but also normal DTC migration to form wild-type Ushaped gonad arms in $v a b-10(t k 27)$ animals (Fig. 6C-E). The rate of DTC migration relative to the vulval development was also restored (see Fig. S2C in the supplementary material). Because coexpression of ABD-Venus and MTBD-Venus failed to rescue the DTC defects in $v a b-10(t k 27)$ animals, it is likely that ABD and MTBD must be connected for rescue to occur (Fig. 6B). ABDMTBD-Venus showed filamentous signals both in the wild-type and vab-10(tk27) mutant backgrounds (Fig. 6F,G). These observations suggest that ABD-MTBD-Venus can serve as a functional substitute for endogenous VAB-10B1.

\section{DISCUSSION}

\section{Structures and expression of VAB-10B1 isoforms}

Spectraplakin family genes produce complex sets of isoforms by using alternative splicing and alternative promoters (Roper et al., 2002). The $C$. elegans vab-10 gene also produces a number of splicing isoforms that are grouped into two major classes: VAB-10A and VAB-10B (Bosher et al., 2003). The promoter element upstream of exon 1 and the one in intron 5 are likely to transcribe mRNAs for VAB-10A1 and $\mathrm{B} 1$ and $\mathrm{VAB}-10 \mathrm{~A} 2$ and $\mathrm{B} 2$, respectively. Immunohistochemical analysis revealed that VAB-10A is expressed in the epidermis, whereas VAB-10B is distributed in several tissues, including DTCs and the epidermis. In this study, we showed that there are at least two functionally different $\mathrm{VAB}-10 \mathrm{~B}$ isoforms: VAB-10B1 and VAB-10B2. VAB-10B1, which has a complete ABD containing both calponin homology $1(\mathrm{CH} 1)$ and $\mathrm{CH} 2$ domains, was required for proper DTC migration, whereas VAB-10B2, which contains only the $\mathrm{CH} 2$ domain, was required for epidermal morphogenesis in embryonic development. In Drosophila Shot, the first $\mathrm{CH}$ domain is also dispensable for epidermal functions (Bottenberg et al., 2009). Spectraplakins, which have two, one or no $\mathrm{CH}$ domains, are produced by alternative splicing in mammals, and these N-terminal variations are suggested to alter the interaction with F-actin: $\mathrm{CH} 1$ and $\mathrm{CH} 2$ together bind strongly, $\mathrm{CH} 1$ binds weakly and $\mathrm{CH} 2$ does not bind F-actin (Gimona et al., 2002; Lee and Kolodziej, 2002a). Therefore, VAB-10B1 and VAB-10B2 may differ in their affinity for F-actin.

\section{VAB-10B1 is required for organization of actin and MT cytoskeleton within DTCs}

VAB-10B1 (but not VAB-10A and VAB-10B2) was expressed in the DTCs of wild-type animals and was absent from vab-10(tk27) animals, suggesting that VAB-10B1 expressed in the DTCs plays roles to achieve normal DTC migration and the nuclear migration. In ACF7-deficient endodermal cells and keratinocytes, the radial MT projection was severely disrupted, whereas the F-actin network was mostly unaffected (Kodama et al., 2003; Wu et al., 2008). We also observed that the MT network, but not the F-actin network, was severely disorganized in the vab-10(tk27) mutant DTCs. The fact that the DTC-specific expression of ABD-MTBD-Venus (vab10 mini-gene) significantly rescued the mutant defects indicates that ABD and MTBD must be connected to substitute for VAB$10 \mathrm{~B} 1$ activity. Because the rescue was partial, it is, however, possible that $\mathrm{VAB}-10 \mathrm{~B} 1$ has roles other than acting as a bridge between F-actin and MTs in DTCs. The lack of the EF-hand domain (discussed below) in the ABD-MTBD-Venus construct used in this work might be a part of the reason for partial rescue. The linking function of spectraplakins for F-actin and MTs has 

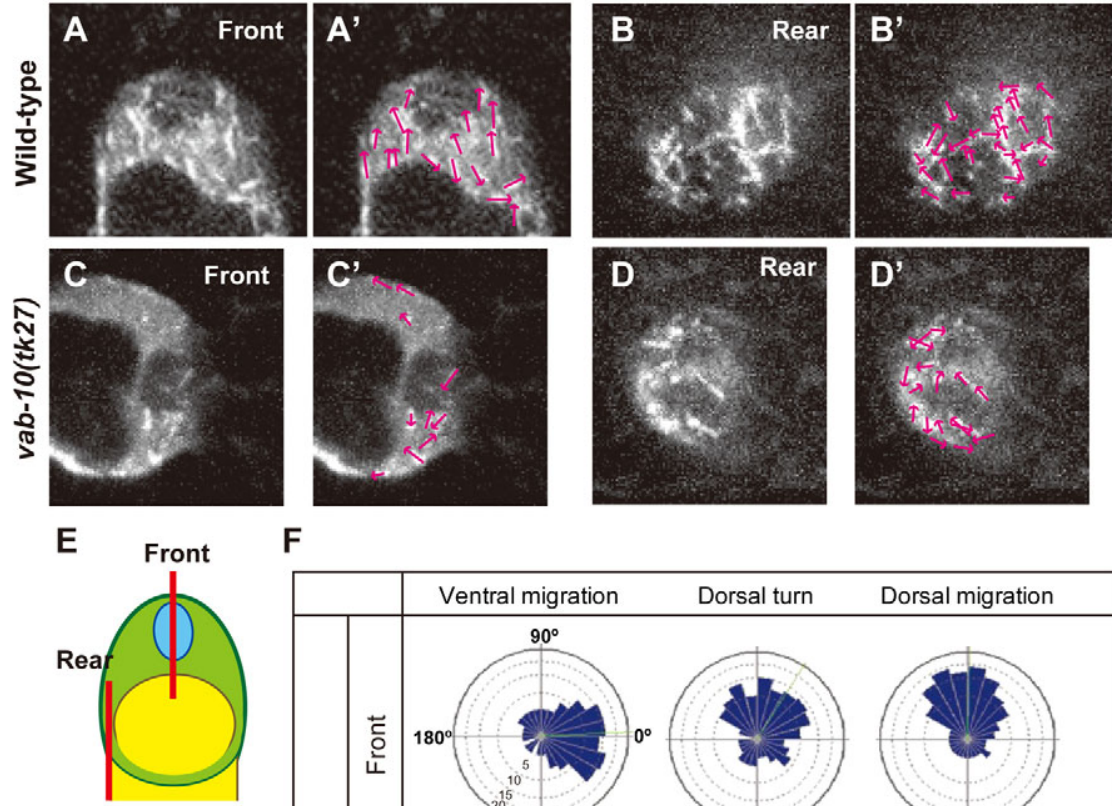

$\mathbf{F}$

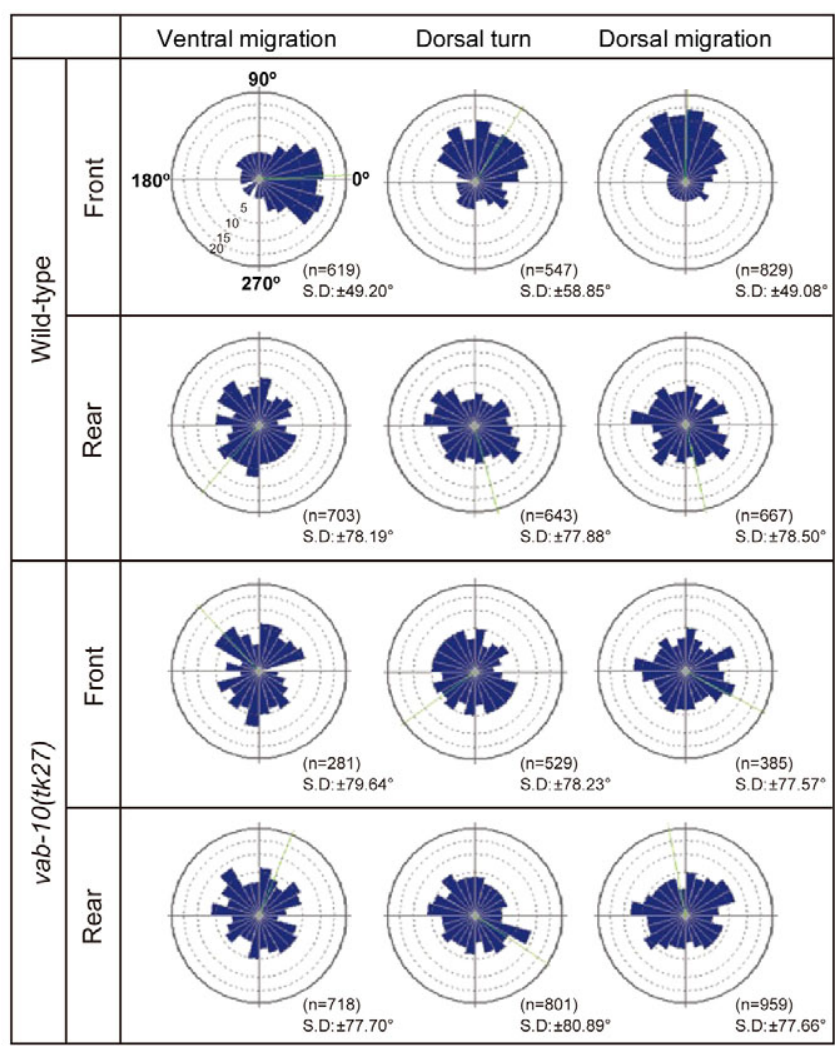

Fig. 5. Direction of MT growth in DTCs. EBP-2GFP comets were tracked and their directions were analyzed. (A-D') Images representing 4 seconds of 200 mseconds/frame of EBP-2-GFP time-lapse sequences of the front $(A, C)$ and rear $(B, D)$ regions of wild-type $(A, B)$ and vab-10(tk27) $(C, D)$ DTCs that are migrating dorsally. 'Front' is the nuclear side of the cell and 'rear' is the opposite side. (E) Schematic presentation of the planes (shown in bars) of front and rear optical sections analyzed in $A-D^{\prime}$. The direction of each shooting comet in A-D is indicated by arrows $\left(A^{\prime}-D^{\prime}\right)$. (F) Directional distribution of comets in DTCs on the ventral muscle (ventral migration), at the dorsal turn and during dorsal migration is shown by Rose diagrams composed of 24 bins of $15^{\circ}$ each. $0^{\circ}, 90^{\circ}, 180^{\circ}$ and $270^{\circ}$ correspond to distal, dorsal, proximal and ventral directions, respectively. The concentric circles were drawn with 5\% increments between them. Green lines represent the average angles. been reported in Drosophila Shot and the mammalian ACF7, both of which link F-actin and MTs through their direct binding or through indirect binding via MT plus-end tracking proteins (+TIPs) (Slep et al., 2005; Subramanian et al., 2003; Wu et al., 2008). It is not known whether $C$. elegans spectraplakins bind +TIPs.

Our analysis using a $C$. elegans +TIP, EBP-2, revealed dynamic polarized outgrowth of MTs in the migrating DTCs. The number and directional movement of EBP-2-GFP comets in the front region of DTCs were affected in $t k 27$, but the randomized movement of comets in the rear region was not. Thus, VAB-10B1 plays important roles in the outgrowth of MTs in the front region of DTCs. As VAB-10B1 is expressed at this location, it is tempting to speculate that VAB-10B1 could stabilize MT + end and/or promote MT growth by interacting with EBP-2. Interestingly, Shot, the Drosophila spectraplakin, has been reported to organize and maintain a compact MT-rich domain, possibly by recruiting EB1 to the muscle/tendon junction (Subramanian et al., 2003).

\section{Spectraplakin VAB-10B1 has a crucial role in nuclear migration}

Because DTCs in $t k 27$ animals migrate more slowly than those in the wild type and this phenotype was rescued by expression of the vab-10 mini-gene containing only $\mathrm{ABD}$ and MTBD, it is clear that VAB-10B1 is required for a normal rate of migration. The mutant DTCs can, however, make two turns and even migrate halfway to the final stopping position on the dorsal muscle. Thus, VAB-10B1 and the polarized outgrowth of MTs in the front region are not essential for turning and migration of DTCs. It is possible that the F-actin network, which is not severely affected by the $t k 27$ allele, might cover this essential function in DTC migration. The weak extension of lamellipodium in $t k 27$ mutants might be the indirect consequence of microtubule misregulation. Alternatively, it could be the direct consequence of loss of VAB-10B1 functions in actin regulation, analogous to findings for Drosophila Shot (Dilks and DiNardo, 


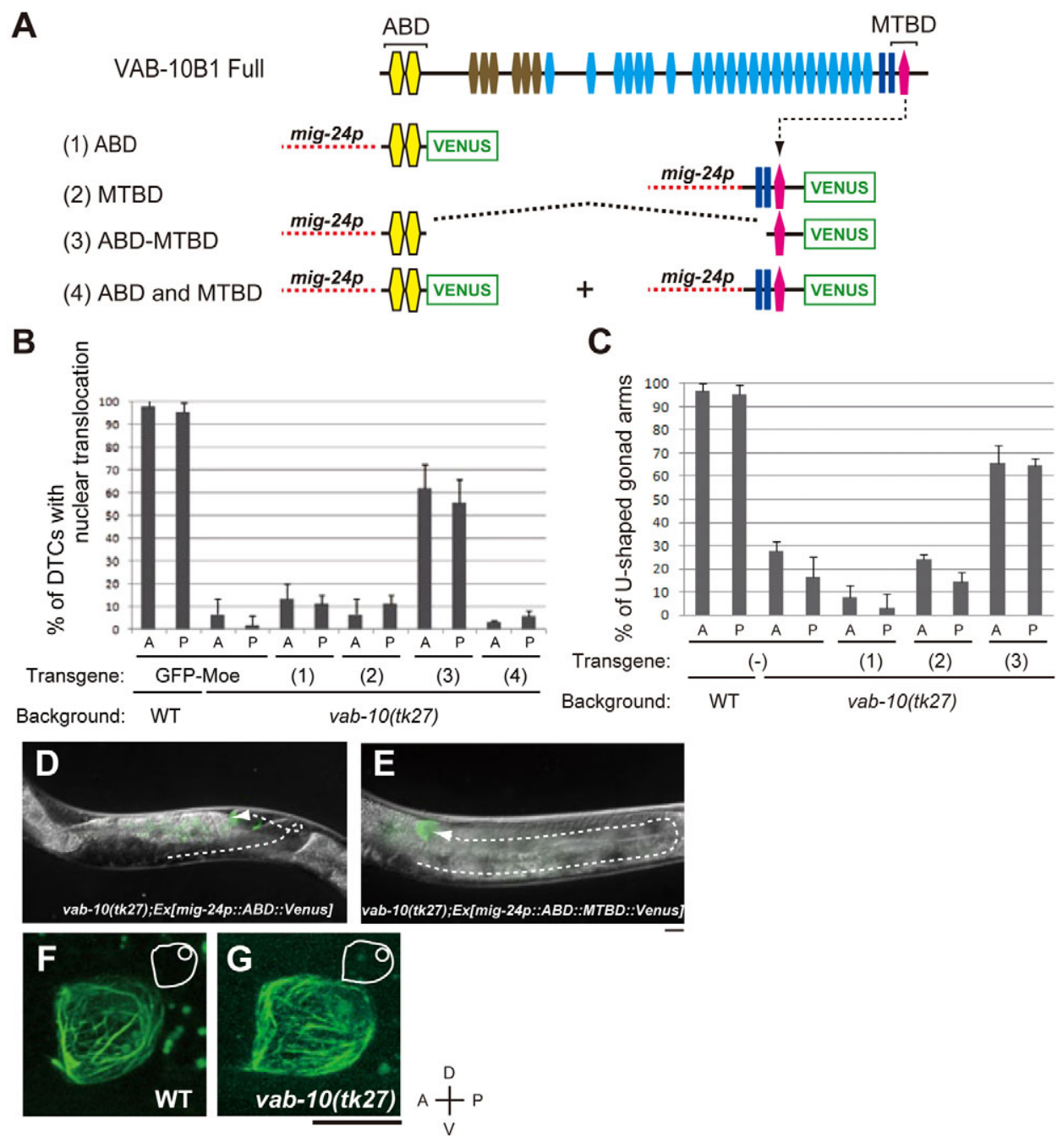

Fig. 6. Rescue experiments with vab-10(tk27) using deletion constructs of VAB-10B1. (A) Schematic diagrams of constructs used in rescue experiments. Transgenic vab-10(tk27) worms carrying extrachromosomal arrays containing (1) mig-24p::ABD::Venus, (2) mig-24p::MTBD::Venus, (3) mig-24p::ABD-MTBD::Venus and (4) both mig-24p::ABD::Venus and mig-24p::MTBD::Venus were generated. (B) The percentage of DTCs that underwent nuclear translocation at the first turn in various transgenic worm strains is shown. The numbers in parentheses correspond to those for the constructs in A. Anterior (A) and posterior (P) DTCs were scored independently. The data are shown as the mean \pm s.d. ( $n=45$ for each experiment). (C) The percentage of anterior and posterior gonad arms that adopted the U-shaped morphology. The numbers in parentheses correspond to those of the constructs in A. The data are shown as the mean \pm s.d. ( $n=45$ for each experiment). (D,E) Representative animals of vab10(tk27); Ex[mig-24p::ABD::Venus] (D) and vab-10(tk27); Ex[mig-24p::ABD-MTBD::Venus] (rescued) (E). The deduced trajectories of DTCs are shown by broken arrows. (F,G) Z-stack confocal images (0.5 $\mu \mathrm{m}$ intervals) of ABD-MTBD-Venus in the wild type (F) and in vab-10(tk27) animals (G). DTCs that had undergone nuclear translocation are shown. The DTCs and their nuclei are illustrated schematically with white lines in each image. Anterior is leftwards and dorsal is upwards. Scale bars: $10 \mu \mathrm{m}$.

2010; Lee et al., 2007; Sanchez-Soriano et al., 2009), where the EF-hand domain is essential for actin regulation. VAB-10B1 also has an EF-hand domain in its $\mathrm{C}$ terminus (Fig. 1K).

In contrast to the mild defect in DTC cell migration, nuclear migration was strikingly impaired in $t k 27$. Therefore, the ability to move the DTC nucleus is strongly dependent on VAB-10B1. Spectraplakins are also involved in the structuring, positioning and organization of organelles, including the nucleus, endoplasmic reticulum and mitochondria in other systems (Gupta et al., 2010; Roper and Brown, 2004; Young et al., 2003; Young et al., 2006; Young and Kothary, 2008). Because the MT outgrowth in the DTC front region has a clear polarity that directs the plus ends towards the nucleus, it is possible that MTs play roles in nuclear translocation of DTCs. The MT outgrowth in the front part of DTCs migrating on the ventral muscle is mostly from proximal to distal, whereas the directionality is gradually shifted to from ventral to dorsal during the first turn, indicating that the MT alignment in DTCs is actively remodeled during the turning process. Because this regulated MT outgrowth was lost in $t k 27$ animals, it is likely that VAB-10B1 has a crucial role in the formation and remodeling of the MT network by which the nucleus is translocated during redirection of the DTCs.

It has been reported that nuclear migration in the embryonic epidermal hyp7 cells is mediated by kinesin, dynein and SUN and KASH proteins in C. elegans (Fridolfsson and Starr, 2010). The $\mathrm{KASH}$ protein interacts with the SUN protein in the nuclear 


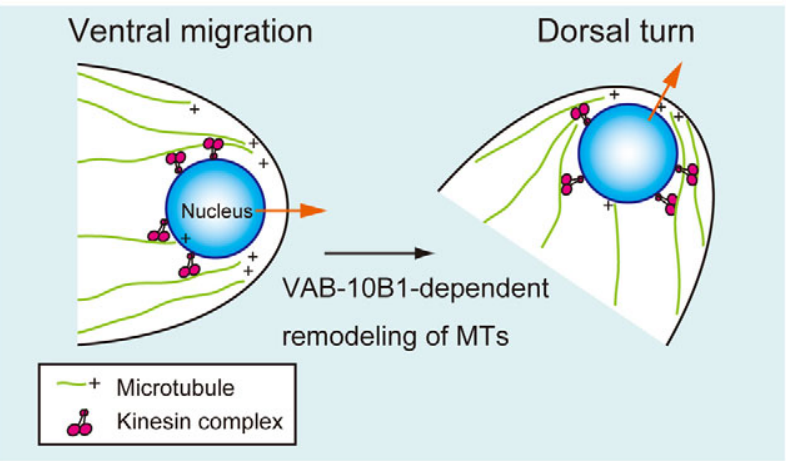

Fig. 7 Model for nuclear translocation in DTCs. In the front region of DTCs, VAB-10B1 acts to regulate the outgrowth of MTs to direct the plus ends towards the migratory leading edge of the cell. The nuclei of DTCs are carried to the plus ends of arrayed MTs by kinesindependent manner (orange arrows). During dorsal turn of DTCS, VAB$10 \mathrm{~B} 1$ is required for MT remodeling to shift the direction of MT outgrowth.

membrane and recruits kinesin to regulate nuclear migration (Starr, 2009). In this system, the nuclei are carried from minus to plus ends along the MT filaments. We also observed that nuclear translocation in DTCs is both kinesin and KASH dependent (H.-S.K. and K.N., unpublished). Therefore, it might be possible that the nuclei in the DTCs continue to be carried to the plus ends of arrayed MT fibers, the direction of which is gradually changed in a VAB-10B1dependent manner during the first turn of the DTCs. This function of VAB-10B1 probably facilitates keeping nuclei at the leading edge of the DTCs during the course of migration (Fig. 7). Although we observed a strong nuclear translocation defect similar to that in $v a b$ 10(tk27) mutants in the unc-83 (encoding a KASH protein) mutants, DTC migration in these mutants was affected only weakly (H.-S.K. and K.N., unpublished), suggesting that nuclear translocation itself does not play a major role in cell migration of the DTCs.

In conclusion, our data suggest that the spectraplakin VAB-10B1 has important functions in nuclear translocation and cell migration of DTCs that involve regulating the polarized outgrowth of MTs. This mechanism appears to require VAB-10B1 activity to link Factin and MTs. Our findings provide the first evidence of the function of spectraplakin in directed nuclear migration and the involvement of spectraplakin in the tip cell-dependent regulation of epithelial tube morphogenesis. Our finding also raises the interesting possibility that the function of spectraplakin might be especially important in the tip-cell(s) of growing epithelial tubes during organogenesis.

\section{Acknowledgements \\ We thank Asako Sugimoto, Hitoshi Sawa and the members of our laboratory for their helpful discussions. We also thank Shigeo Hayashi for the moesin ABD plasmid and Martin Srayko for the EBP-2-GFP plasmid. Some nematode strains were provided by the Caenorhabditis Genetics Center, which is funded by the $\mathrm{NIH}$ National Center for Research Resources (NCRR). This work was supported by a Grant-in-Aid for Scientific Research on Innovative Areas to K.N. Deposited in PMC for release after 12 months.}

\section{Competing interests statement}

The authors declare no competing financial interests.

\section{Supplementary material}

Supplementary material for this article is available at

http://dev.biologists.org/lookup/suppl/doi:10.1242/dev.059568/-/DC1

\section{References}

Bosher, J. M., Hahn, B. S., Legouis, R., Sookhareea, S., Weimer, R. M., Gansmuller, A., Chisholm, A. D., Rose, A. M., Bessereau, J. L. and Labouesse, M. (2003). The Caenorhabditis elegans vab-10 spectraplakin isoforms protect the epidermis against internal and external forces. J. Cell Biol. 161, 757-768.

Bottenberg, W., Sanchez-Soriano, N., Alves-Silva, J., Hahn, I., Mende, M. and Prokop, A. (2009). Context-specific requirements of functional domains of the Spectraplakin Short stop in vivo. Mech. Dev. 126, 489-502.

Brenner, S. (1974). The genetics of Caenorhabditis elegans. Genetics 77, 71-94.

Caussinus, E., Colombelli, J. and Affolter, M. (2008). Tip-cell migration controls stalk-cell intercalation during Drosophila tracheal tube elongation. Curr. Biol. 18, 1727-1734.

Culotti, J. G. and Merz, D. C. (1998). DCC and netrins. Curr. Opin. Cell Biol. 10, 609-613.

Dalpe, G., Leclerc, N., Vallee, A., Messer, A., Mathieu, M., De Repentiqny, Y. and Kothary, R. (1998). Dystonin is essential for maintaining neuronal cytoskeleton organization. Mol. Cell. Neurosci. 10, 243-257.

Dilks, S. A. and DiNardo, S. (2010). Non-cell-autonomous control of denticle diversity in the Drosophila embryo. Development 137, 1395-1404.

Edwards, K. A., Demsky, M., Montague, R. A., Weymouth, N. and Kiehart, D. P. (1997). GFP-moesin illuminates actin cytoskeleton dynamics in living tissue and demonstrates cell shape changes during morphogenesis in Drosophila. Dev. Biol. 191, 103-117.

Finney, M. and Ruvkun, G. (1990). The unc-86 gene product couples cell lineage and cell identity in C. elegans. Cell 63, 895-905

Fridolfsson, H. N. and Starr, D. A. (2010). Kinesin-1 and dynein the nuclear envelope mediate the bidirectional migrations of nuclei. J. Cell Biol. 191, 115128

Gimona, M., Djinovic-Carugo, K., Kranewitter, W. J. and Winder, S. J. (2002). Functional plasticity of $\mathrm{CH}$ domains. FEBS Lett. 513, 98-106.

Goryunov, D., He, C. Z., Lin, C. S., Leung, C. L. and Liem, R. K. (2010). Nervous-tissue-specific elimination of microtubule-actin crosslinking factor 1a results in multiple developmental defects in the mouse brain. Mol. Cell. Neurosci. 44, 1-14.

Guo, L., Degenstein, L., Dowling, J., Yu, Q. C., Wollmann, R., Perman, B. and Fuchs, E. (1995). Gene targeting of BPAG1: abnormalities in mechanical strength and cell migration in stratified epithelia and neurologic degeneration. Cell 81, 233-243.

Gupta, T., Marlow, F. L., Ferriola, D., Mackiewicz, K., Dapprich, J., Monos, D. and Mullins, M. C. (2010). Microtubule actin crosslinking factor 1 regulates the Balbiani body and animal-vegetal polarity of the zebrafish oocyte. PLOS. Genet. 6, e1001073.

Harumoto, T., Ito, M., Shimada, Y., Kobayashi, T. J., Ueda, H. R., Lu, B. and Uemura, T. (2010). Atypical cadherins Dachsous and Fat control dynamics of noncentrosomal microtubules in planar cell polarity. Dev. Cell 19, 389-401.

Jefferson, J. J., Leung, C. L. and Liem, R. K. (2006). Dissecting the sequence specific functions of alternative $\mathrm{N}$-terminal isoforms of mouse bullous pemphigoid antigen 1. Exp. Cell Res. 312, 2712-2725.

Karakesisoglou, I., Yang, Y. and Fuchs, E. (2000). An epidermal plakin that integrates actin and microtubule networks at cellular junctions. J. Cell Biol. 149, 195-208.

Kodama, A., Karakesisoglou, I., Wong, E., Vaezi, A. and Fuchs, E. (2003). ACF7: an essential integrator of microtubule dynamics. Cell 115, 343-354.

Kubota, Y., Sano, M., Goda, S., Suzuki, N. and Nishiwaki, K. (2006). The conserved oligomeric Golgi complex acts in organ morphogenesis via glycosylation of an ADAM protease in C. elegans. Development 133, 263-273.

Lee, S. and Kolodziej, P. A. (2002a). Short Stop provides an essential link between F-actin and microtubules during axon extension. Development 129, 1195-1204.

Lee, S. and Kolodziej, P. A. (2002b). The plakin Short Stop and the RhoA GTPase are required for E-cadherin-dependent apical surface remodeling during tracheal tube fusion. Development 129, 1509-1520.

Lee, S., Nahm, M., Lee, M., Kwon, M., Kim, E., Zadeh, A. D., Cao, H., Kim, H. J., Lee, Z. H., Oh, S. B. et al. (2007). The F-actin-microtubule crosslinker Shot is a platform for Krasavietz-mediated translational regulation of midline axon repulsion. Development 134, 1767-1777.

Leung, C. L., Sun, D., Zheng, M., Knowles, D. R. and Liem, R. K. (1999). Microtubule actin cross-linking factor (MACF): a hybrid of dystonin and dystrophin that can interact with the actin and microtubule cytoskeletons. J. Cell Biol. 147, 1275-1286.

Lu, P. and Werb, Z. (2008). Patterning mechanisms of branched organs. Science 322, 1506-1509.

Maduro, M. and Pilgrim, D. (1995). Identification and cloning of unc-119, a gene expressed in the Caenorhabditis elegans nervous system. Genetics 141, 977-988.

Mello, C. C., Kramer, J. M., Stinchcomb, D. and Ambros, V. (1991). Efficient gene transfer in C.elegans: extrachromosomal maintenance and integration of transforming sequences. EMBO J. 10, 3959-3970. 
Meyerzon, M., Fridolfsson, H. N., Ly, N., McNally, F. J. and Starr, D. A. (2009). UNC-83 is a nuclear-specific cargo adaptor for kinesin-1-mediated nuclear migration. Development 136, 2725-2733.

Nagai, T., Ibata, K., Park, E. S., Kubota, M., Mikoshiba, K. and Miyawaki, A. (2002). A variant of yellow fluorescent protein with fast and efficient maturation for cell-biological applications. Nat. Biotechnol. 20, 87-90.

Roper, K. and Brown, N. H. (2004). A spectraplakin is enriched on the fusome and organizes microtubules during oocyte specification in Drosophila. Curr. Biol. 14, 99-110

Roper, K., Gregory, S. L. and Brown, N. H. (2002). The 'spectraplakins': cytoskeletal giants with characteristics of both spectrin and plakin families. J. Cell Sci. 115, 4215-4225.

Sainio, K., Suvanto, P., Davies, J., Wartiovaara, J., Wartiovaara, K., Saarma, M., Arumae, U., Meng, X., Lindahl, M., Pachnis, V. et al. (1997). Glial-cellline-derived neurotrophic factor is required for bud initiation from ureteric epithelium. Development 124, 4077-4087.

Sanchez-Soriano, N., Travis, M., Dajas-Bailador, F., Goncalves-Pimentel, C., Whitmarsh, A. J. and Prokop, A. (2009). Mouse ACF7 and drosophila short stop modulate filopodia formation and microtubule organisation during neuronal growth. J. Cell Sci. 122, 2534-2542.

Shakya, R., Watanabe, T. and Costantini, F. (2005). The role of GDNF/Ret signaling in ureteric bud cell fate and branching morphogenesis. Dev. Cell 8, 6574.

Slep, K. C., Rogers, S. L., Elliott, S. L., Ohkura, H., Kolodziej, P. A. and Vale, R. D. (2005). Structural determinants for EB1-mediated recruitment of APC and spectraplakins to the microtubule plus end. J. Cell Biol. 168, 587-598.

Sonnenberg, A. and Liem, R. K. (2007). Plakins in development and disease. Exp. Cell Res. 313, 2189-2203.
Srayko, M., Kaya, A., Stamford, J. and Hyman, A. A. (2005). Identification and characterization of factors required for microtubule growth and nucleation in the early C. elegans embryo. Dev. Cell 9, 223-236.

Starr, D. A. (2009). A nuclear-envelope bridge positions nuclei and moves chromosomes. J. Cell Sci. 122, 577-586.

Su, M., Merz, D. C., Killeen, M. T., Zhou, Y., Zheng, H., Kramer, J. M., Hedgecock, E. M. and Culotti, J. G. (2000). Regulation of the UNC-5 netrin receptor initiates the first reorientation of migrating distal tip cells in Caenorhabditis elegans. Development 127, 585-594.

Subramanian, A., Prokop, A., Yamamoto, M., Sugimura, K., Uemura, T. Betschinger, J., Knoblich, J. A. and Volk, T. (2003). Shortstop recruits EB1/APC 1 and promotes microtubule assembly at the muscle-tendon junction. Curr. Biol. 13, 1086-1095.

Sun, D., Leung, C. L. and Liem, R. K. (2001). Characterization of the microtubule binding domain of microtubule actin crosslinking factor (MACF): identification of a novel group of microtubule associated proteins. J. Cell Sci. 114, 161-172.

Tamai, K. K. and Nishiwaki, K. (2007). bHLH transcription factors regulate organ morphogenesis via activation of an ADAMTS protease in C. elegans. Dev. Biol. $308,562-571$

Wu, X., Kodama, A. and Fuchs, E. (2008). ACF7 regulates cytoskeletal-focal adhesion dynamics and migration and has ATPase activity. Cell 135, 137-148.

Young, K. G. and Kothary, R. (2008). Dystonin/Bpag1 is a necessary endoplasmic reticulum/nuclear envelope protein in sensory neurons. Exp. Cell Res. 314, 27502761.

Young, K. G., Pool, M. and Kothary, R. (2003). Bpag1 localization to actin filaments and to the nucleus is regulated by its N-terminus. J. Cell Sci. 116, 4543-4555.

Young, K. G., Pinheiro, B. and Kothary, R. (2006). A Bpag1 isoform involved in cytoskeletal organization surrounding the nucleus. Exp. Cell Res. 312, 121-134. 